\title{
Equivalence of conditional and external field ensembles in absorbing state phase transitions
}

\author{
Gunnar Pruessner \\ Mathematics Institute, University of Warwick, Gibbet Hill Road, Coventry CV4 7AL, UK*
}

\begin{abstract}
I comment on the relation between two sampling methods for absorbing state models. It is shown that a certain ensemble without external field conditional to activity coincides with the unconditional ensemble for sufficiently small external field. The actual physical processes involved are identical and the derivation of the identity of the scaling behaviour relies on a single (established) scaling law. While the conditional ensemble by construction does not contain information about the system with large external field, it contains all information about the limit of vanishing external field and about the vicinity of the critical point: Finite size scaling as well as critical scaling in the temperature-like variable or in (small) external field.
\end{abstract}

\section{INTRODUCTION}

Absorbing state (AS) models suffer from the problem that the activity necessarily ceases in any finite system in the stationarity state [1]. In order to see a phase transition, one cannot naïvely probe the stationary state. Two approaches are widely accepted to overcome this problem: Either an external field is applied that creates spontaneous activity [2] or an ensemble conditional to activity is considered [3] (or technically more sophisticated [4]). Both methods effectively do away with the absorbing state altogether. The additional external field might be physically more appealing because it resolves the problem by introducing an external driving that appears naturally in the corresponding field theories. On the other hand, the external field represents a parameter that needs to be tuned in addition to the temperature-like variable that drives the transition, because certain universal behaviour can be obtained only for sufficiently small field [5]. The conditional ensemble has one parameter less, but requires the selection of "good samples" which might appear unphysical.

The different techniques have created a rift that goes through the AS literature: Some authors use strong words to reject one and support the other method. In the following it is shown, however, that the external field ensemble coincides with a conditional ensemble. The derivation applies to all models where the external field triggers activity in the same way as an initial seed in the conditional ensemble.

None of the two ensembles is superior to the other, provided they are implemented appropriately. In fact, one can use the same implementation and simply derive one from the other. Although the relation between the two ensembles appears to be rather trivial, it has significant implications in particular for the external field ensemble. Yet, so far, it does not seem to have entered into the AS literature.

\section{DERIVATION}

In the following, the relation between the moments of the activity calculated in the different ensembles is derived. The precise definition of the activity depends on the particular model, yet, all that matters is that the system has an order parameter, the activity, the instantaneous value of which vanishes for good if the system is "left unattended", that is, the system hits an absorbing state after some time. An activation mechanism can trigger a spell of activity (an avalanche) and these "seeds" are either implemented as an external field $h$ or as the initialisation in the conditional ensemble.

Firstly, I introduce the $n$th moment of the activity in the external field ensemble, $\left\langle\rho^{n}\right\rangle_{H}$. This is derived from a record of instantaneous activity $\rho_{H}(t)$, see Fig. 1 , in the obvious way,

$$
\left\langle\rho^{n}\right\rangle_{H}=T_{0}^{-1} \int_{0}^{T_{0}} d t \rho_{H}^{n}(t),
$$

where the time $T_{0}$ is the time span of the entire observation.

*Present address: Department of Mathematics, Imperial College London, 180 Queen's Gate, London SW7 2AZ 


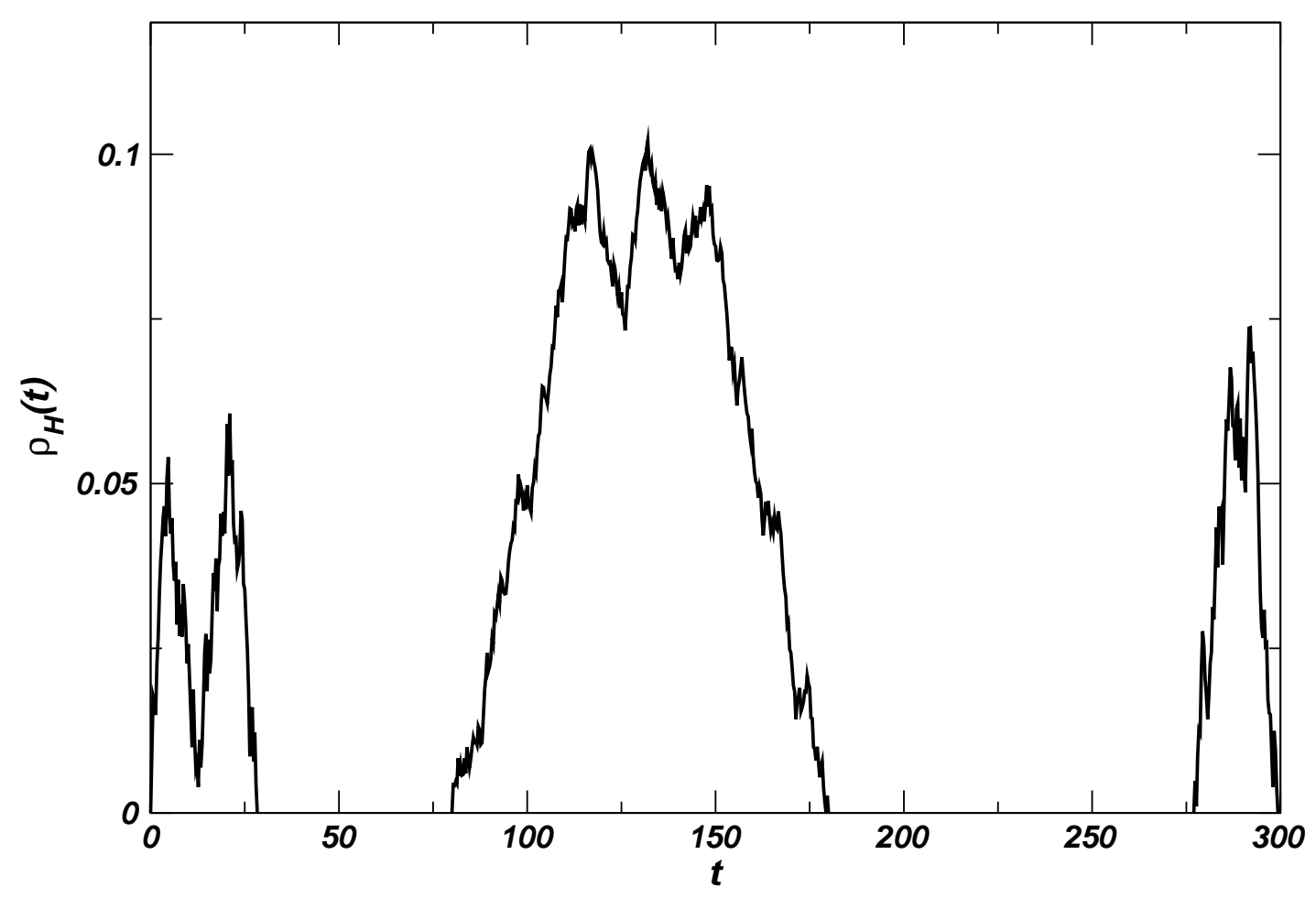

FIG. 1: Cartoon of a continuous record of activity in a system with a small external field $h$ which triggers new avalanches typically with some time of complete quiescence between them. The total time span covered is $T_{0}=300$. The record could have been produced by compiling the three instances shown in Fig. 2.

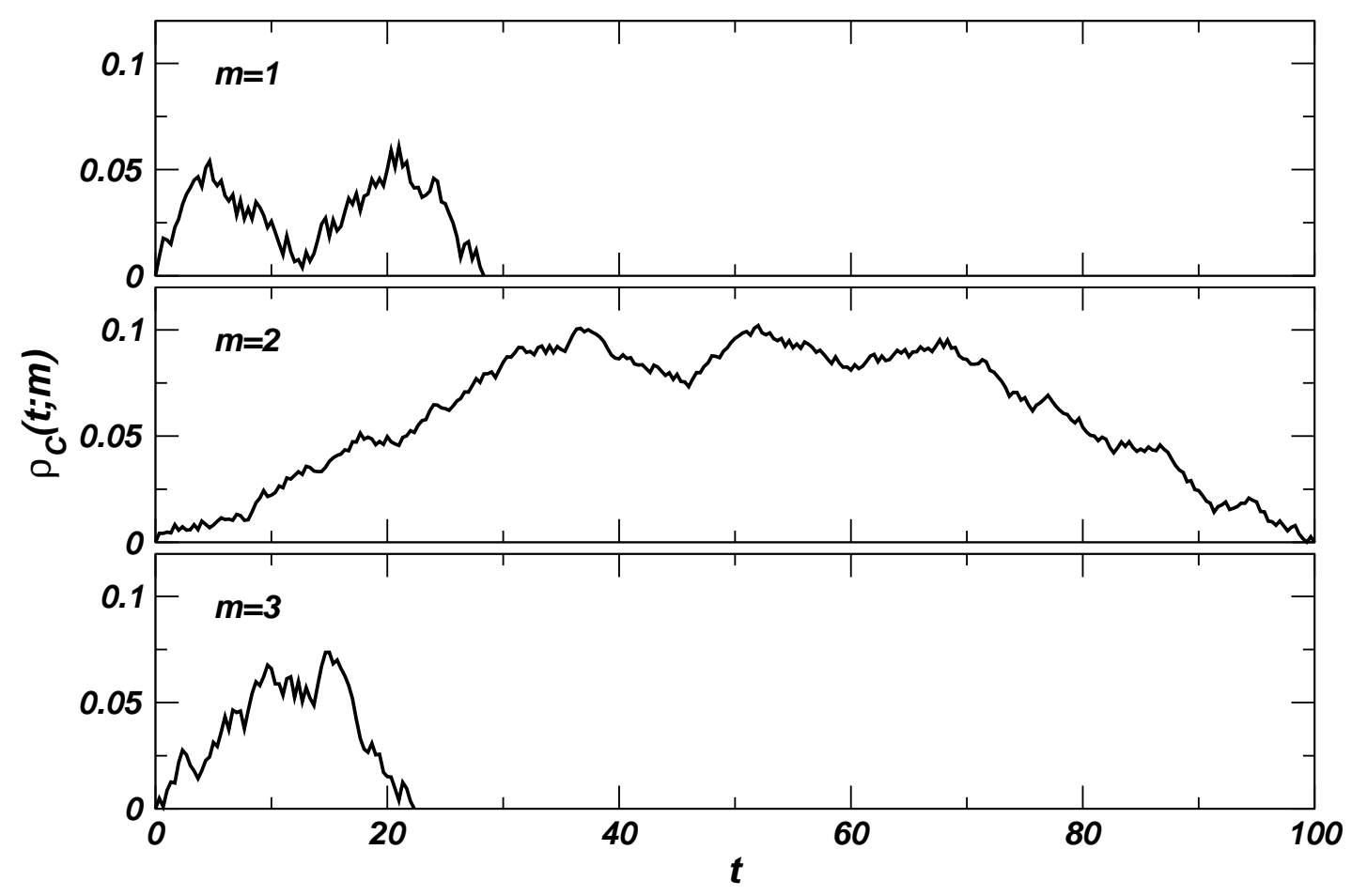

FIG. 2: Cartoon of three avalanches produced by placing "seeds" in an otherwise inactive system. The three instances could have been taken from the continuous record in Fig. 1. 
Secondly, I define the $n$th moment of the activity in the conditional ensemble as the average taken conditional to the system being active at all. Fig. 2 shows three instances of conditional activity. To define it formally, $\rho_{C}(t ; m)$ is the $m$ th instance (i.e. the $m$ th run) of a spell of activity in the system at time $t$ after initialisation at $t=0$. If $t_{m}$ is the time the system is active, the moments are

$$
\left\langle\rho^{n}\right\rangle_{C}=\frac{\sum_{m=1}^{M} \int_{0}^{t_{m}} d t \rho_{C}^{n}(t ; m)}{\sum_{m=1}^{M} t_{m}} .
$$

Two technical remarks: Firstly, one should formally distinguish between the estimate of the average from a finite sample and the ensemble average. For brevity, this is not done here. Secondly, $\left\langle\rho^{n}\right\rangle_{C}$ is often estimated slightly differently to allow for a transient [6]: One defines

$$
\left\langle\rho^{n}\right\rangle_{C}(T)=\frac{\sum_{m=1}^{M} \theta\left(t_{m}-T\right) \int_{T}^{t_{m}} d t \rho_{C}^{n}(t ; m)}{\sum_{m=1}^{M} \theta\left(t_{m}-T\right)\left(t_{m}-T\right)}
$$

which is a temporal average from $t=T$ to $t=\infty$ of the average (across instances) instantaneous activity conditional to activity, weighted by the number of systems active at time $t$. A transient can then be established as the time $T_{0}$ after which the average $\left\langle\rho^{n}\right\rangle_{C}(T)$ has reached a (quasi-)stationary state (see discussion below). Transients are commonly used in equilibrium statistical mechanics, to suppress the effect of the initialisation. In the following, no transient is discriminated, i.e. $\left\langle\rho^{n}\right\rangle_{C}=\left\langle\rho^{n}\right\rangle_{C}(T=0)$ is used.

Both, $\left\langle\rho^{n}\right\rangle_{H}$ and $\left\langle\rho^{n}\right\rangle_{C}$ depend on the system size $L$ and $\left\langle\rho^{n}\right\rangle_{H}$ is, in addition, a function of the field $h$. However, the records $\rho_{C}(t)$ can be derived from $\rho_{H}(t)$ and vice versa, provided the field $h$ is small enough. This is illustrated in Fig. 1 and Fig. 2: The three records in the latter Figure are derived from the former, by restarting the clock $t$ after the $m$ th spell of activity is triggered in $\rho_{H}(t)$. On the other hand, given an ensemble of avalanches $\rho_{C}(t ; m)$, a record $\rho_{H}(t)$ can be constructed by introducing gaps of vanishing activity between spells of activity taken from $\rho_{C}(t ; m)$. Without knowing whether the $\rho_{C}(t ; m)$ are independent for different $m$, they have to be compiled sequentially. This property, however, will not be used in the following.

To construct the basic observables $\rho_{H}(t)$ from $\rho_{C}(t ; m)$ correctly, one would also need to know the distribution of waiting times. This can, in principle, depend on the configuration of the system and might therefore vary if the system has more than one absorbing state. However, in the following only the average waiting time will enter.

If the two observables are derived from each other in this way, one has by construction

$$
\left\langle\rho^{n}\right\rangle_{H}=T_{0}^{-1} \int_{0}^{T_{0}} d t \rho_{H}^{n}(t)=T_{0}^{-1} \sum_{m=1}^{M} \int_{0}^{t_{m}} d t \rho_{C}^{n}(t ; m)=\frac{\sum_{m=1}^{M} t_{m}}{T_{0}}\left\langle\rho^{n}\right\rangle_{C} .
$$

For a large number of avalanches $M$ the pre-factor $T_{0}^{-1} \sum_{m=1}^{M} t_{m}$ converges to $\left\langle w_{m}\right\rangle_{H}^{-1}\left\langle t_{m}\right\rangle_{C}$ where $\left\langle w_{m}\right\rangle_{H}$ is the average time that passes between two avalanches being triggered in the external field ensemble and $\left\langle t_{m}\right\rangle_{C}$ is the average duration of an avalanche.

In order to derive $\left\langle w_{m}\right\rangle_{H}$ given $h$ one needs to know how the external field is implemented. Provided it operates as a Poisson process throughout the system, one has $\left\langle w_{m}\right\rangle_{H}=(V h)^{-1}$ where $V$ is the volume of the system and $h$ is the field (density) or flux. It is also clear that the mechanism to trigger avalanches used in the conditional ensemble must correspond to the external field operating in the system with field. This scenario is the case for the vast majority of models considered in absorbing state phase transitions. This is particularly obvious if only one absorbing state is present, so that single "seeds", which arrive independently, uniformly and with constant rate everywhere in the system, trigger avalanches.

Accepting the Poissonian nature of the external field, one arrives at the central result

$$
\left\langle\rho^{n}\right\rangle_{H}(\delta p, h ; L)=h V(L)\left\langle t_{m}\right\rangle_{C}(\delta p ; L)\left\langle\rho^{n}\right\rangle_{C}(\delta p ; L),
$$

valid asymptotically for sufficiently small field. In Eq. (5) all dependences of the individual observables on the parameters have been stated explicitly. The linearity in small field of all momenta $\left\langle\rho^{n}\right\rangle_{H}(\delta p, h ; L) \propto h$ is consistent with the literature, e.g. [5] but see [13]. The parameter $\delta p$ is the temperature-like variable that drives a transition at $\delta p=0$, subject of the following considerations.

\section{DISCUSSION}

The above construction requires the field $h$ to be so small that no new seeds arrive while an avalanche is running, or, more specifically, that the statistical weight of these events is negligible. Otherwise $\left\langle\rho^{n}\right\rangle_{C}$ derived from $\left\langle\rho^{n}\right\rangle_{H}$ 
would depend on the external field, which has been excluded in Eq. (5) and is crucial for the following derivations. In other words, the derivation relies on a separation of time scales; in this sense $\left\langle\rho^{n}\right\rangle_{C}$ is an observable as would be used in Self-Organised Criticality [7], while $\left\langle\rho^{n}\right\rangle_{H}$ represents the "instantaneous activity" [8, 9]

Therefore, Eq. (5) can only be expected to hold if $(V h)^{-1}$ is large compared to $\left\langle t_{m}\right\rangle_{C}$. This is the case for sufficiently small field at given system size, i.e. in particular for finite size scaling where the system size is fixed before the limit $h \rightarrow 0$ is taken. The condition is also met in the inactive phase, $\delta_{p}<0$, where the average duration of the avalanches converges with increasing system size, so that, again, the average duration can be chosen to be small compared to the time scale set by the external field. In this case, the thermodynamic limit can be taken before $h \rightarrow 0$. However, in the active phase, the average duration diverges extremely fast with system size, so that the external field can never be sufficiently small when the thermodynamic limit is taken first.

Because the external field $h$ appears only as a pre-factor on the RHS of Eq. (5), it is very simple to derive the scaling of $\left\langle\rho^{n}\right\rangle_{H}(\delta p, h ; L)$ from the scaling of $\left\langle t_{m}\right\rangle_{C}(\delta p ; L)$ and $\left\langle\rho^{n}\right\rangle_{C}(\delta p ; L)$ assuming, naturally, $V(L)=a_{V} L^{d}$, where $d$ is the spatial dimension of the system. The converse, i.e. the derivation of the scaling of $\left\langle\rho^{n}\right\rangle_{C}(\delta p ; L)$ from the scaling of $\left\langle\rho^{n}\right\rangle_{H}(\delta p, h ; L)$, is not as simple and therefore shown explicitly in the following.

Standard scaling assumptions suggest [5]

$$
\left\langle\rho^{n}\right\rangle_{H}(\delta p, h ; L)=a_{n} \lambda^{-\beta_{n}} R_{n}^{H}\left(a_{p} \delta p \lambda, a_{h} h \lambda^{\sigma}, a_{L} L \lambda^{-\nu_{\perp}}\right)
$$

with non-universal metric factors [10] $a_{n}, a_{p}$ etc., arbitrary (scaling) parameter $\lambda$, scaling function $R_{n}^{H}$ and the usual critical exponents $\sigma$ (field exponent), $\nu_{\perp}$ (spatial correlation length exponent) and $\beta_{n}$, the latter being $\beta_{1}=\beta$ (order parameter exponent) for the first moment and $\beta_{n}=n \beta$ if gap-scaling applies [11]. Comparing Eq. (6) to Eq. (5) implies that $R_{n}^{H}$ is asymptotically linear in the (small) external field

$$
R_{n}^{H}\left(a_{p} \delta p, a_{h} h, a_{L} L\right)=a_{h} h \tilde{R}_{n}\left(a_{p} \delta p, a_{L} L\right),
$$

where $\tilde{R}_{n}$ must obey Eq. (6) as well, i.e. $\tilde{R}_{n}\left(a_{p} \delta p, a_{L} L\right)=\lambda^{\sigma-\beta_{n}} \tilde{R}_{n}\left(a_{p} \delta p \lambda, a_{L} L \lambda^{-\nu_{\perp}}\right)$.

Eq. (7) implies that the susceptibility $\chi_{H}(\delta p, h ; L)=\frac{\partial\langle\rho\rangle_{H}}{\partial h}$, or the derivative of any moment $\left\langle\rho^{n}\right\rangle_{H}$ with respect to $h$, is independent from the external field under the conditions stated earlier.

To derive the scaling of $\left\langle\rho^{n}\right\rangle_{C}(\delta p ; L)$, one needs the scaling of $\left\langle t_{m}\right\rangle_{C}(\delta p, L)$ which is well understood [5],

$$
\left\langle t_{m}\right\rangle_{C}(\delta p, L)=a_{t} T\left(a_{p} \delta p, a_{L} L\right)=a_{t} \lambda^{\nu_{\|}(1-\delta)} T\left(a_{p} \delta p \lambda, a_{L} L \lambda^{-\nu_{\perp}}\right)
$$

where $\nu_{\|}$is the temporal correlation length exponent, $\delta$ is the survival exponent and $\nu_{\|}(1-\delta)=\sigma-d \nu_{\perp}$, which will be crucial for the next step. Combining Eqns. (5), (6), (7) and (8) produces the scaling the scaling of the conditional moments:

$$
\left\langle\rho^{n}\right\rangle_{C}(\delta p ; L)=\frac{a_{h} a_{n} a_{L}^{d}}{a_{V} a_{t}} \lambda^{-\beta_{n}} R_{n}^{C}\left(a_{p} \delta p \lambda, a_{L} L \lambda^{-\nu_{\perp}}\right),
$$

where $R_{n}^{C}(x, y)=\tilde{R}_{n}(x, y) /\left(y^{d} T(x, y)\right)$.

Comparing Eq. (9) to Eq. (6) shows that both ensembles display the same scaling behaviour. The lack of this proof might explain the critical remarks about the conditional ensemble in [2]. The finite size scaling for the two observables, however, differs: Setting $\delta p=0$ and choosing $\lambda$ so that $a_{L} L \lambda^{-\nu_{\perp}}=1$ Eq. (9) gives

$$
\left\langle\rho^{n}\right\rangle_{C}(0 ; L)=\frac{a_{h} a_{n} a_{L}^{d-\beta_{n} / \nu_{\perp}}}{a_{V} a_{t}} L^{-\beta_{n} / \nu_{\perp}} R_{n}^{C}(0,1) .
$$

Because $\left\langle\rho^{n}\right\rangle_{C}(0 ; L)$ is non-zero by definition but bound from above, $R_{n}^{C}(0,1)$ must be finite, so that $\left\langle\rho^{n}\right\rangle_{C}(0 ; L) \propto$ $L^{-\beta_{n} / \nu_{\perp}}$ as one would expect from equilibrium critical phenomena. Similarly, one finds

$$
\left\langle\rho^{n}\right\rangle_{H}(0, h ; L)=a_{n} a_{L}^{-\beta_{n} / \nu_{\perp}} L^{-\beta_{n} / \nu_{\perp}} R_{n}^{H}\left(0, a_{h} h\left(a_{L} L\right)^{\sigma / \nu_{\perp}}, 1\right)
$$

$$
=a_{n} a_{L}^{-\beta_{n} / \nu_{\perp}} L^{-\beta_{n} / \nu_{\perp}} a_{h} h\left(a_{L} L\right)^{\sigma / \nu_{\perp}} \tilde{R}_{n}(0,1)
$$

from Eq. (6) and Eq. (7). Again, the activity $\left\langle\rho^{n}\right\rangle_{H}(0, h ; L)$ is bound from above and does not vanish at finite external field, so that $\tilde{R}_{n}(0,1)$ is finite, and therefore $\left\langle\rho^{n}\right\rangle_{H}(0, h ; L) \propto L^{\left(\sigma-\beta_{n}\right) / \nu_{\perp}}$. This rather unusual finite size scaling behaviour of the activity is documented in the literature [5] (in particular Figures 33 and 47). The effective finite size scaling exponent for $n=1$ is $(\sigma-\beta) / \nu_{\perp}=\gamma / \nu_{\perp}$, which is the finite size scaling exponent of the susceptibility. Indeed, for small external fields at $\delta p=0$ and in finite systems, one expects $\langle\rho\rangle_{H}=h \chi_{H}$. 
Eq. (5) implies that, under the general conditions stated earlier, all moments vanish linearly in the external field. The Binder cumulant

$$
Q(\delta p, h, L)=1-\frac{\left\langle\rho^{4}\right\rangle_{H}}{3\left\langle\rho^{2}\right\rangle_{H}^{2}}
$$

therefore diverges like $h^{-1}$. In [2] it has been suggested that a divergent Binder cumulant is a "characteristic feature of all absorbing phase transitions", but in the light of the above derivation, it appears rather like a generic feature of the particular ensemble. Moreover, replacing $\left\langle\rho^{n}\right\rangle_{H}$ by the conditional averages $\left\langle\rho^{n}\right\rangle_{C}$ in Eq. (12) renders the Binder cumulant a universal moment ratio again and reinstates it as a "very useful method" to identify the transition. The same applies to lower moment ratios, which can be determined with higher accuracy, such as $\langle\rho\rangle_{C}^{2} /\left\langle\rho^{2}\right\rangle_{C}$.

It is now also clear why the slightly more complicated moment ratio proposed in [12]

$$
U=\frac{\left\langle\rho^{2}\right\rangle_{H}\left\langle\rho^{3}\right\rangle_{H}-\langle\rho\rangle_{H}\left\langle\rho^{2}\right\rangle_{H}^{2}}{\langle\rho\rangle_{H}\left\langle\rho^{4}\right\rangle_{H}-\langle\rho\rangle_{H}\left\langle\rho^{2}\right\rangle_{H}^{2}}
$$

converges to a finite value: Both, denominator as well as numerator are, to leading order, quadratic in $h$. In fact

$$
\lim _{h \rightarrow 0} U(\delta p, h, L)=\lim _{h \rightarrow 0} \frac{\left\langle\rho^{2}\right\rangle_{H}\left\langle\rho^{3}\right\rangle_{H}}{\langle\rho\rangle_{H}\left\langle\rho^{4}\right\rangle_{H}}=\frac{\left\langle\rho^{2}\right\rangle_{C}\left\langle\rho^{3}\right\rangle_{C}}{\langle\rho\rangle_{C}\left\langle\rho^{4}\right\rangle_{C}}
$$

where the first equality holds because $\langle\rho\rangle_{H}\left\langle\rho^{2}\right\rangle_{H}^{2} \in \mathcal{O}\left(h^{3}\right)$, and the second because the remaining terms carry the same prefactor, namely $\left(h V(L)\left\langle t_{m}\right\rangle_{C}(\delta p ; L)\right)^{2}$, see Eq. (5). The findings above are also confirmed by the exact results in [12] (the RHS of Eq. (21) in [12] should read 2/( $\pi x)$ for $x \rightarrow 0$ ).

The linearity of $\langle\rho\rangle_{H}$ in $h$, Eq. (7), contradicts the mean field result $\langle\rho\rangle_{H}(\delta p=0, h)=\sqrt{h}[5]$, which is, however, consistent with mean field theory not allowing a proper distinction between conditional ensemble and external field ensemble.

The limitations of Eq. (5) become clearer when comparing to the standard scaling assumption $\lim _{L \rightarrow \infty}\langle\rho\rangle_{H}(\delta p=$ $0, h ; L) \propto h^{\beta / \sigma}[1]$. Eq. (5) seems to suggest that $\beta / \sigma=1$ which clearly is not the case. However, Eq. (5) applies only where the waiting time $(h V)^{-1} \propto L^{-d}$ is large compared to $\left\langle t_{m}\right\rangle_{C} \propto L^{\sigma / \nu_{\perp}-d}$, which cannot be the case if $\sigma / \nu_{\perp}>0$ and the thermodynamic limit taken at finite field $h$. It is worth noting, however, that Eq. (7) relies solely on the Poissonian nature of the external field.

Finally, I want to discuss briefly two features of conditional ensembles as they often appear in the literature, namely the initial condition and the discounting of a transient. The latter was introduced as $T$ in Eq. (3), by defining the conditional $n$th moment as an average taken from $t=T$ to $t \rightarrow \infty$. For any non-vanishing transient $T>0$ the strict identity of conditional and external field ensemble breaks down. To restore the above results, more elaborate arguments are needed, based on the length of transient, its contribution to the average and its scaling.

Similarly, there is no strict identity of the two ensembles, if the conditional ensemble is based on initial conditions which do not correspond to configurations reached when the external field operates on inactive configurations in the external field ensemble. On the other hand, one might hope that the introduction of a transient erases the dependence on the initial condition and that asymptotically, the effect of the transient and the initial condition becomes negligible.

\section{SUMMARY}

To summarise, I address the issue of the relation between the two ensembles usually considered in absorbing state phase transitions. It turns out that the ensemble of "activity conditional to activity" can be derived from the ensemble obtained by applying an external field and vice versa, provided that the avalanche duration is small compared to the waiting time between two avalanches. Technically, both methods can be implemented in (almost) the same way. Because moment ratios, such as the Binder cumulant, remain finite in the conditional ensemble and because moments of the activity in the presence of an external field all vanish linearly in the external field, the conditional ensemble might be more comfortable to study. 


\section{Acknowledgements}

The author would like to thank Alastair Windus and Sven Lübeck for useful discussions and the RCUK for support.

[1] H. Hinrichsen, Adv. Phys. 49, 815 (2000), cond-mat/0001070v2.

[2] S. Lübeck and P. C. Heger, Phys. Rev. E 68, 056102 (2003).

[3] J. Marro and R. Dickman, Nonequilibrium Phase Transitions in Lattice Models (Cambridge University Press, New York, 1999).

[4] M. M. de Oliveira and R. Dickman, Phys. Rev. E 71, 016129 (2005).

[5] S. Lübeck, Int. J. Mod. Phys. B 18, 3977 (2004).

[6] R. Dickman, private communication (2003).

[7] H. J. Jensen, Self-Organized Criticality (Cambridge University Press, New York, NY, 1998).

[8] A. Vespignani and S. Zapperi, Phys. Rev. Lett. 78, 4793 (1997).

[9] G. Pruessner and O. Peters, Phys. Rev. E 73, 025106(R) (2006), cond-mat/0411709.

[10] V. Privman, P. C. Hohenberg, and A. Aharony, in Phase Transitions and Critical Phenomena, edited by C. Domb and J. L. Lebowitz (Academic Press, New York, 1991), vol. 14, chap. 1, pp. 1-134.

[11] P. Pfeuty and G. Toulouse, Introduction to the Renormalization Group and to Critical Phenomena (John Wiley \& Sons, Chichester, 1977).

[12] S. Lübeck and H.-K. Janssen, Phys. Rev. E 72, 016119 (2005).

[13] It remains unclear why one of 17 models shown in Figures 33 and 47 of [5] fails to display the linearity. 